\title{
EFETIVIDADE DO PROGRAMA DE COMUNICAÇÃO E EDUCAÇ̃̃O EM SAÚDE NO CONTROLE DA INFECÇÃO POR S. MANSONI EM ALGUMAS ÁREAS DO ESTADO DA BAHIA
}

\author{
Vilma Sousa Santana, Maria da Glória Teixeira, \\ Cristina P. Santos e Charles A.R. de Andrade
}

\begin{abstract}
O Programa de Controle da Esquistossomose (PCE) vem sendo desenvolvido em áreas da Babia pela Fundação Nacional de Saúde (FNS). Em 1989, ações de Informação, Educação, Comunicação e Mobilização Comunitária (IEC/MC) foram iniciadas. Neste estudo avalia-se o impacto epidemiológico do IEC/MC, adotando-se um desenho quasiexperimental, comparando-se prevalências de infecção por S. mansoni em áreas IEC/MC com estimativas de áreas referentes. Os dados são secundários, coletados rotineiramente pela FNS. Verificou-se uma redução da prevalência da esquistossomose em todas as áreas, que alcança maior intensidade nas áreas com IEC/MC. Aparentemente, ações de controle rotineiras realizadas isoladamente são mais efetivas entre escolares e pessoas do sexo masculino, enquanto que nas áreas com IEC/MC, observou-se maior impacto entre as mulheres, refletindo, provavelmente, as distintas estratégias adotadas. Aponta-se para a necessidade de estudos de avaliação qualitativos, além de estimativas do custo-benefício e custo-efetividade de modo a aprimorar o processo de tomada de decisões.
\end{abstract}

Palavras-chaves: Esquistossomose. S. mansoni. Controle. Avaliação. Educação. Tendência temporal.

Programas de controle da esquistossomose têm sido avaliados em estudos que tratam do impacto sobre a prevalência da infecção 317 22, intensidade da infecção13 152028 , formas clínicas 9 , e até mesmo da incidência da infecção e reinfecção18. São raras, todavia, pesquisas sobre aspectos específicos dos processos de intervenção como cobertura, qualidade, ou seus componentes, como as práticas educativas. Nesse trabalho, pretende-se verificar qual o impacto epidemiológico de um programa voltado para a Informação, Educação, Comunicação em Saúde e Mobilização Comunitária, o IEC/MC, desenvolvido pela Fundação Nacional de Saúde em alguns estados brasileiros.

A avaliação do papel da educação, informação e comunicação na prevenção da esquistossomose requer o delineamento dos

\footnotetext{
Instituto de Saúde Coletiva, Universidade Federal da Bahia e Fundação Nacional de Saúde, Regional da Bahia (MS/FNS), Salvador, BA.

Trabalho financiado parcialmente pela Fundação Nacional de Saúde e pelo Conselho Nacional de Desenvolvimento Científico e Tecnológico

Endereço para correspondência: Dr ${ }^{\mathrm{a}}$ Vilma S. Santana. Instituto de Saúde Coletiva/UFBA, R. Padre Feijó 29 , $4^{\circ}$ andar, 40110-170 Salvador, BA, Brasil.

Tel: 55 (071) 245-0544: Fax: 55 (071) 237-5856.

Recebido para publicação em 03/02/97.
}

modelos teóricos de controle que fundamentam suas estratégias. Woolhouse 27 apresenta uma síntese desses modelos, identificando aqueles que visam a erradicação da infecção pela redução do número médio de $S$. mansoni por pessoa, até um limiar necessário para que a infecção residual se extinga, o que descartaria a necessidade de intervenções para a sua manutenção; a redução sustentada da infecção também poderia levar à uma diminuição da taxa de reprodução basal do parasito, em níveis abaixo dos quais manter-se-ia a erradicação. Quanto ao controle voltado apenas para a redução da infecção, sua estratégia objetiva a queda do número médio de $S$. mansoni por pessoa, da prevalência de quadros sintomáticos e de infectados com alta carga parasitária, os quais estariam associados à ocorrência da enfermidade esquistossomótica, em um determinado período de tempo. A quimioterapia, embora apresente um impacto instantâneo na redução da infecção, requer ciclos repetidos em pequenos intervalos de tempo, de modo a permitir a interrupção da transmissão.

Achados de estudos avaliativos sugerem que apenas o saneamento básico e o abastecimento d'água seriam alternativas factíveis de controle, os quais poderiam determinar a redução 

em Saúde no controle da infecção por S. mansoni em algumas áreas do Estado da Babia. Revista da Sociedade Brasileira de Medicina Tropical 30:447-456, nov-dez, 1997.

sustentada da taxa de contaminação que integra a transmissão homem-caramujo15 27. Modificações comportamentais também poderiam atuar como redutoras da reinfecção, através da alteração dos padrões de contato com água contaminada e, conseqüentemente, reduzindo a taxa de transmissão homem-caramujo. Todavia, alterações de comportamento humano são um desafio para a saúde pública, especialmente quando se trata de enfermidades que acometem populações rurais com acesso limitado aos meios de comunicação de massa e baixa escolaridade. Ademais, a água integra aspectos comportamentais relacionados com o prazer, a sobrevivência e até mesmo a fé religiosa, os quais demarcam resistências especiais à intervenção. Tem sido observado que a água está envolvida no lazer (banhos de rio)19, no trabalho doméstico (lavagem de roupas ou utensílios em rios ou córregos), atividades ocupacionais (pesca, caça ou plantio) e práticas religiosas como o batismo em algumas religiões 51426 , embora nem sempre venham sendo alvo de abordagens adequadas nos programas de controle.

A associação de atividades diversificadas, onde a quimioterapia ocupa papel primordial, se constitui no eixo estratégico dos programas de controle da esquistossomose implementados no Brasil11. Entretanto, a avaliação do impacto da quimioterapia seletiva ou de massa tem mostrado resultados pouco animadores para a erradicação e até para a redução da infecção, restringindo-se os seus efeitos à redução da intensidade da infecção 27 . Do ponto de vista teórico, em geral, os modelos elaborados para os programas de controle tomam as mudanças comportamentais como elemento essencial da sustentabilidade do efeito instantâneo da quimioterapia, pois estão voltados para a redução dos contatos ou do tempo de exposição com as águas contaminadas com ovos de S. mansoni, como também para a alteração dos padrões de disposição das excretas 15. Pesquisas avaliativas sobre o controle da esquistossomose não têm contemplado especificamente o componente educação em saúde16. Barbosa6, em uma avaliação de um programa de controle que incorporava educação sanitária associada a medidas de saneamento básico e abastecimento d'água, verificou uma redução da prevalência da infecção esquistossomótica em um período de sete anos, mesmo sem o uso da quimioterapia ou de moluscicidas.

O Programa Especial de Controle da Esquistossomose (PECE), desde sua origem previa o desenvolvimento de atividades de educação em saúde11. Adotava-se um modelo tradicional calcado no repasse de conhecimento pelos guardas sanitários da SUCAM/FNS. Isto se processava em visitas domiciliares, utilizandose, de modo padronizado, descrição oral das formas de transmissão e meios de prevenir a doença11. A incorporação do componente IEC ao Programa de Controle das Doenças Endêmicas do Nordeste (PCDEN) a partir de 1989, propunha uma nova pedagogia, na qual o conhecimento formal era confrontado com o "conhecimento vivencial" das comunidades na tentativa de se construir participativamente novas representações e práticas 24 . Essas práticas, de acordo com as diretrizes do Ministério da Saúde12, se opunham à uma "concepção estática de educação, entendida apenas como transferência de conhecimentos, habilidades e destreza", assumindo uma metodologia de participação que capacitaria indivíduos e grupos para a transformação da realidade. Desse modo, estratégias integradas de informação, educação, comunicação em saúde e mobilização comunitária em torno dessas ações modificariam comportamentos e práticas, viabilizando a proteção à doenças e a luta pelo direito à saúde e, não somente a participação passiva em um conjunto de ações previamente definido. Em 1994, após cinco anos de investimentos no programa IEC/MC, a FNS realizou um estudo multicêntrico de avaliação da efetividade dessas ações no controle de endemias, integrando antropologia à tradicional abordagem epidemiológica. Este é o componente epidemiológico desse estudo, que foi realizado em alguns municípios do Estado da Bahia, através da comparação de medidas de impacto populacional. A esquistossomose mansônica foi a endemia escolhida pela sua importância na saúde pública e disponibilidade dos dados secundários que viabilizaram a realização da pesquisa.

\section{MATERIAL E MÉTODOS}

Esse é um estudo quasi-experimental baseado em dados de prevalência seriada da esquistossomose mansônica, obtidos em dois pontos do tempo, em áreas com programa IEC/MC e uma outra região onde apenas o programa de controle de rotina da FNS, o PCE, encontrava-se em desenvolvimento. As áreas do estudo foram escolhidas baseando-se nos seguintes critérios: a) existência e disponibilidade 
Santana VS, Teixeira MG, Santos CP, Andrade CAR. Efetividade do Programa de Comunicacão e Educação em Saúde no controle da infecção por S. mansoni em algumas áreas do Estado da Bahia. Revista da Sociedade Brasileira de Medicina Tropical 30:447-456, nov-dez, 1997.

de dados individuais de carga parasitária em pelo menos dois pontos no tempo, de acordo com informações dos servidores da FNS/SUCAM; b) prevalência de infecção acima de $10 \%$ no ponto de tempo inicial; c) informação pela equipe do Distrito Sanitário da FNS sobre a ocorrência de atividades IEC/MC. Selecionouse a sede do município de Boanova e a localidade de Torta como áreas onde haviam sido incorporadas ações IEC/MC às atividades de rotina do PCE. O município de S. Francisco do Conde (22 localidades rurais e áreas do município sede) foi a área de referência, porque nele apenas ações de rotina eram realizadas. Boanova abriga 13.463 habitantes que dispõem de tratamento de água. Nesse local, apenas a população da sede municipal foi examinada. Como Torta é uma localidade do município de Jequié, dados demográficos e sobre sua situação sanitária não estavam disponíveis. São Francisco do Conde é um município com 20.238 habitantes, dispondo de abastecimento de água e esgotamento sanitário através de fossas sépticas e rede de esgoto na área urbana4.

As atividades de rotina da FNS abrangiam um levantamento espacial domiciliar e o censo da população inicial. Posteriormente, coletavamse amostras para exames coprológicos pelo método Kato-Katz. Todos os casos positivos para $S$. mansoni recebiam tratamento quimioterápico, na presença do guarda sanitário (oxamniquine nas doses de $15 \mathrm{mg} / \mathrm{kg}$ para adultos e $20 \mathrm{mg} / \mathrm{kg}$ para crianças até 15 anos de idade). Dados da ficha PCE - 102 sobre o município, localidade, número da casa, nome e idade de todos os habitantes de cada domicílio, número de ovos por grama de fezes, peso, tratamento, dose recebida e data foram registrados.

Dados sobre ações do IEC/MC foram obtidos de Relatórios Técnicos das equipes locais 12 e compreendiam a realização de feiras de saúde, palestras, discussões em reuniões com a comunidade e a distribuição de cartilhas, folhetos e materiais audiovisuais. Ações IEC/MC desenvolvidas no período de 1990 a 1994 em Boa Nova e Torta foram oficinas pedagógicas sobre saúde com ênfase na esquistossomose, utilização de teatros de bonecos e peças teatrais, produção de cartazes, faixas, cenários, divulgação e a elaboração de poesias e repentes. Feiras de saúde foram realizadas com o propósito de disseminação de informações sobre o ciclo de transmissão e medidas de prevenção da doença. Os resultados dos inquéritos coprológicos das localidades foram divulgados e apresentados em palestras para informar sobre as áreas de maior risco, destacando-se a importância da participação popular para a prevenção da infecção. A programação e execução das atividades foram realizadas conjuntamente com as organizações sociais locais, instituições de saúde, de educação e outros campos de atuação social. Professores, alunos e dirigentes das escolas desenvolviam atividades de educação em saúde de diversas modalidades. Para tal, não só introduziam-se conteúdos relativos às formas de prevenção da doença nos currículos escolares, como também realizavam-se atividades especiais extra e intra classes nas escolas de educação formal. Registra-se também que as discussões eram estimuladas, buscando-se sugestões para novas práticas de educação em saúde, de modo a aprimorar a apreensão do conhecimento e buscar formas de estimular o envolvimento de outros segmentos da comunidade. Não foram obtidos relatos sobre a avaliação da aprendizagem e mudanças de práticas ou comportamentos pela população, seja de cuidados individuais ou coletivos de proteção contra a esquistossomose.

Estimaram-se as prevalências da infecção por S. mansoni, geral e de acordo com o nível de intensidade da infecção, em cada uma das áreas e anos do estudo. A carga parasitária era registrada em três níveis, de acordo com o número de ovos por lâmina examinada, I (1 a 4), II (5 a 33) e III (mais de 33). Devido aos pequenos números, as duas últimas faixas foram agrupadas. Variações proporcionais das prevalências (VPP) foram estimadas para o intervalo de tempo (1990 e 1994) de duração das medidas de controle globais e específicas por idade e sexo. Este indicador tem fórmula semelhante ao Risco Atribuível Proporcional, $\mathrm{VPP}=\left(\mathrm{P}_{0}-\mathrm{P}_{1}\right) / \mathrm{P}_{0}$, onde $\mathrm{P} 0$ é a prevalência ao início e $\mathrm{P}_{1}$ ao final do período, empregando-se a prevalência em vez da incidência. Os bancos de dados foram gerados com o EPIINFO 6.0 e a análise realizada com o SAS 6.11 (SAS, 1990). Não se realizaram testes estatísticos devido ao caráter censitário dos inquéritos e à natureza descritiva e exploratória do estudo.

\section{RESULTADOS}

Em Boanova, no primeiro ano do estudo, $1990\left(\mathrm{~T}_{0}\right)$, foram examinadas 357 pessoas em uma área da sede do município, enquanto que em $1994\left(T_{1}\right)$, incluíram-se 3074 pessoas que representavam a totalidade da população. $\mathrm{Na}$ 
Santana VS, Teixeira MG, Santos CP, Andrade CAR. Efetividade do Programa de Comunicacão e Educação em Saúde no controle da infecção por S. mansoni em algumas áreas do Estado da Babia. Revista da Sociedade Brasileira de Medicina Tropical 30:447-456, nov-dez, 1997.

localidade de Torta, em 1990, apenas 129 pessoas participaram do inquérito, e em 1994, 284 indivíduos. Em S. Francisco do Conde, área sem ações IEC/MC, foram realizados três inquéritos: o primeiro, em 1990 quando 1067 pessoas foram examinadas, todas residentes em uma área do município sede; o segundo, em 1994, incluiu 370 pessoas residentes em outras áreas do município sede e em localidades rurais; o terceiro, realizado em 1995 , compreende o re-exame na população das mesmas localidades do segundo inquérito. Para esta área, considerou-se como $\mathrm{T}_{0} \mathrm{O}$ inquérito de 1994, enquanto $\mathrm{T}_{1}$ referiu-se ao ano de 1995.
Impacto sobre a prevalência da infecção por S. mansoni. Na população geral, as prevalências iniciais de infecção por $S$. mansoni variaram de $14,6 \% \mathrm{em} \mathrm{S}$. Francisco do Conde, a 15,4\% em Boanova e 81,5\% em Torta. De acordo com a Figura 1, observa-se redução dessa prevalência em todas as áreas do estudo, seja aquelas com IEC/MC ou São Francisco do Conde que contava apenas com ações de rotina do PCE. A maior queda percentual da prevalência, nos quatro anos do estudo, ocorreu em Boanova, com valor estimado de $85,1 \%$ (de 15,4 para 2,3\%). Em Torta, outra área com IEC/MC, onde era maior

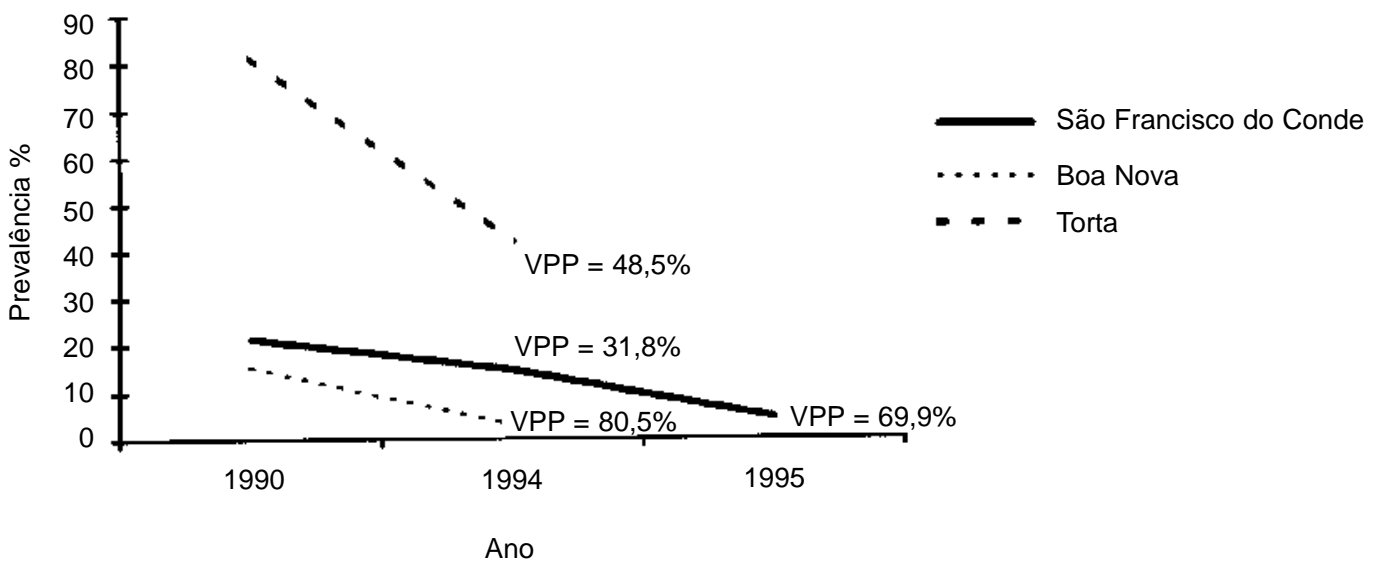

Figura 1 - Variação da prevalência da infecção por S. mansoni nas áreas do estudo por ano da série.

a prevalência inicial, a $\mathrm{VPP}_{\mathrm{pg}}$ foi mais baixa, alcançando apenas $48,5 \%$ (de 81,5 para $42,0 \%)$. Na área de referência, S. Francisco do Conde, estimou-se em 69,9\% a VPP pg (de 14,6 para $4,4 \%)$.

As reduções da prevalência da infecção por $S$. mansoni se diferenciavam de acordo com as faixas de idade, sexo e área. A queda da prevalência foi menos abrupta na população de 7 a 14 anos (Figura 2) em comparação com a população total (Figura 1) nas áreas com IEC $/ \mathrm{MC}$ (Boanova com $\mathrm{VPP}_{\mathrm{e}}=58,3 \%$ e Torta com $\left.\mathrm{VPP}_{\mathrm{e}}=34,9 \%\right)$. Ocorreu o inverso na área controle (S. Francisco do Conde), onde os escolares responderam melhor às ações do PCE $\left(\mathrm{VPP}_{\mathrm{e}}=79,1 \%\right)$ do que a população geral $\left(\mathrm{VPP}_{\mathrm{e}}=69,9 \%\right)$. A evolução da prevalência da infecção por S. mansoni de acordo com o sexo pode ser vista nas Figuras 3 e 4. Não foram identificadas diferenças nas VPP estimadas para os sexos feminino e masculino em Boanova. Em Torta, observou-se um impacto levemente maior entre as mulheres $\left(\mathrm{VPP}_{\mathrm{m}}=34,8 \%\right)$ do que entre os homens $\left(\mathrm{VPP}_{\mathrm{h}}=28,8 \%\right)$. Em S. Francisco do Conde, ao contrário, o impacto foi consideravelmente mais favorável entre os homens $\left(\mathrm{VPP}_{\mathrm{h}}=92,3 \%\right)$ do que entre as mulheres $\left(\mathrm{VPP}_{\mathrm{m}}=57,0 \%\right)$.

Impacto sobre a carga parasitária. Os dados disponíveis sobre carga parasitária foram muito reduzidos em relação aos existentes para a infecção. Consistentemente com os dados brutos relativos à infecção, as estimativas de VPP' ${ }_{p g}$ específicas por nível de carga parasitária sugerem que a maior redução de infectados ocorreu em Torta, área com IEC/MC que deteve uma VPP de 100\%, seja para a população geral ou para os escolares, homens ou mulheres, na 
Santana VS, Teixeira MG, Santos CP, Andrade CAR. Efetividade do Programa de Comunicacão e Educação em Saúde no controle da infecção por S. mansoni em algumas áreas do Estado da Bahia. Revista da Sociedade Brasileira de Medicina Tropical 30:447-456, nov-dez, 1997.

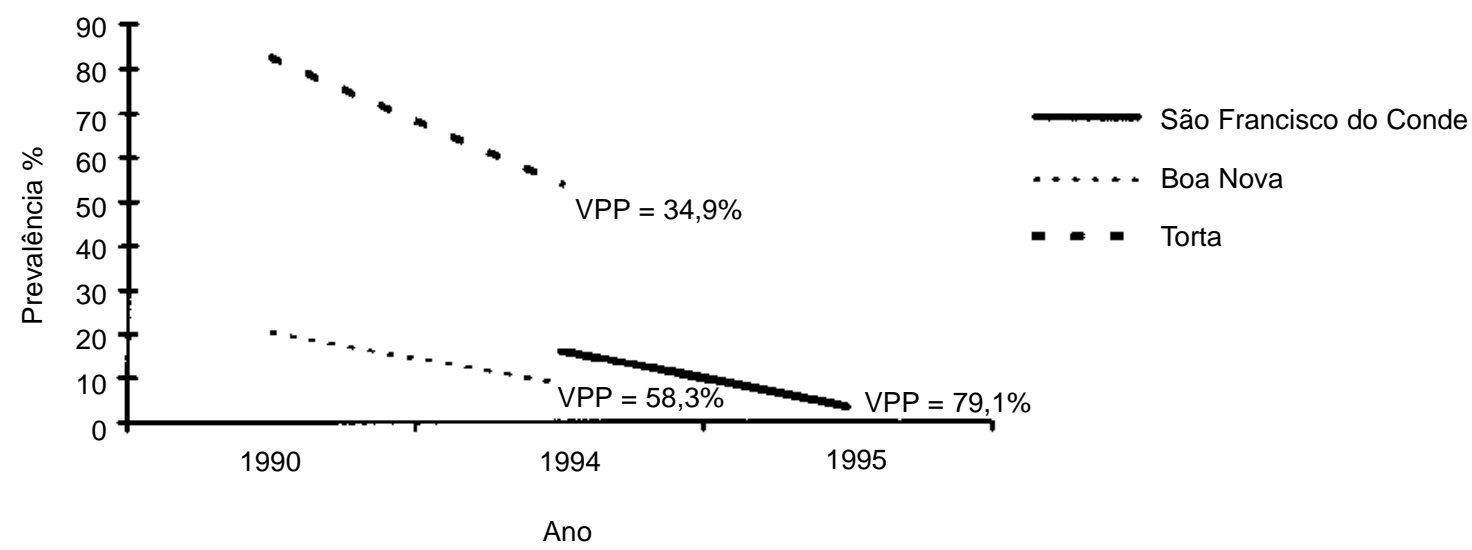

Figura 2 - Variação da prevalência da infecção por S. mansoni nas áreas do estudo por ano da série, população de 7 a 14 anos.

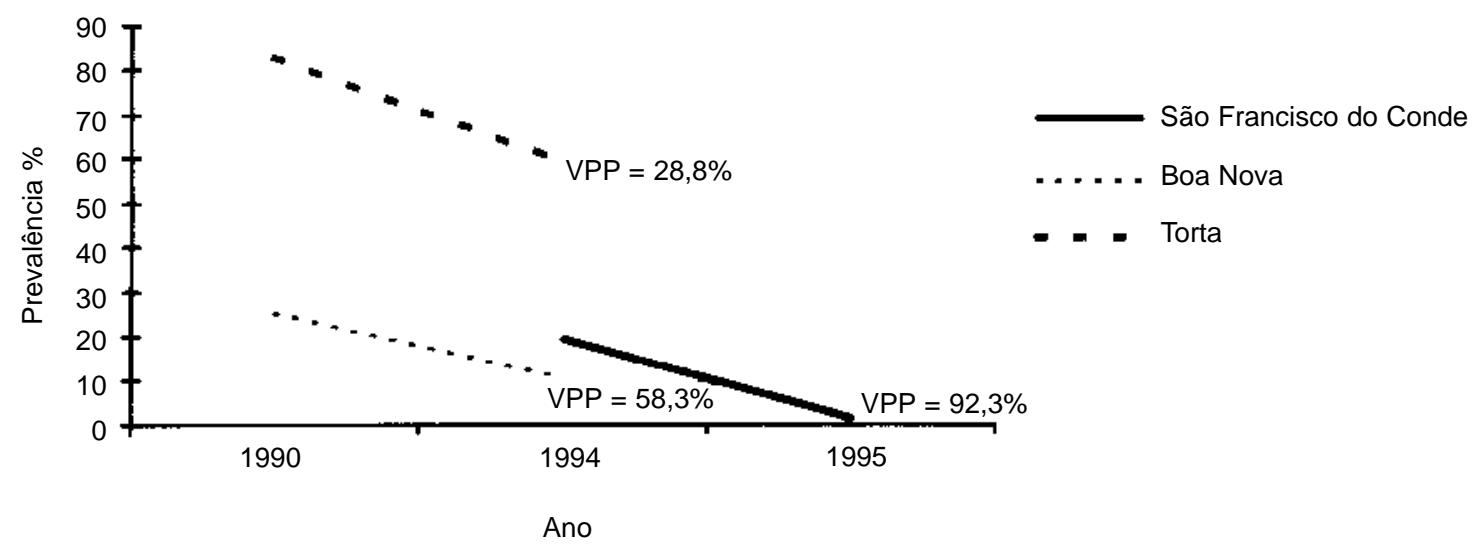

Figura 3 - Variação da prevalência da infecção por S. mansoni em bomens nos anos do estudo.

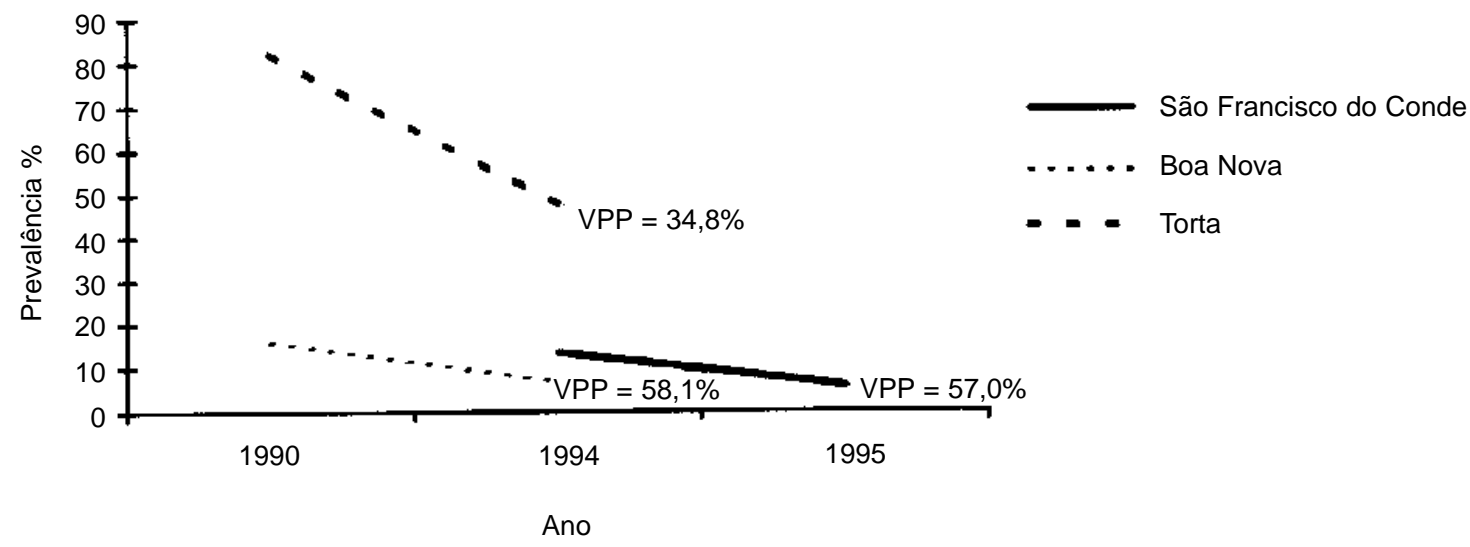

Figura 4 - Prevalência da infecção por S. mansoni em mulheres nos anos do estudo. 
Santana VS, Teixeira MG, Santos CP, Andrade CAR. Efetividade do Programa de Comunicacão e Educação em Saúde no controle da infecção por S. mansoni em algumas áreas do Estado da Babia. Revista da Sociedade Brasileira de Medicina Tropical 30:447-456, nov-dez, 1997.

faixa de 1 a 4 ovos por lâmina (Tabela 1). Ou seja, à época do re-exame, não se identificou nenhum caso nessa faixa de intensidade de infecção. Em Boanova, os resultados mostramse menos marcantes, embora identifiquem-se VPP' abaixo das estimadas para a população de referência (sem o programa) na maioria dos grupos em comparação. Na área sem programa, S. Francisco do Conde, verificaram-se também quedas acentuadas da prevalência para ambas categorias de carga parasitária, com VPP'pg maior $(84,2 \%)$ nos indivíduos de baixa intensidade em comparação com os de alta intensidade de infecção (VPP'pg $=71,4 \%$ )

Tabela 1 - Prevalência da infecção por S. mansoni de acordo com o nível de carga parasitária nas áreas do estudo, todas as idades, por ano da série.

\begin{tabular}{|c|c|c|c|c|c|c|}
\hline \multirow{3}{*}{ Área do estudo } & \multicolumn{6}{|c|}{ Nível da carga parasitária } \\
\hline & \multicolumn{3}{|c|}{1 a 4 ovos/lâmina } & \multicolumn{3}{|c|}{$>4$ ovos/lâmina } \\
\hline & $\mathrm{T}_{0}$ & $\mathrm{~T}_{1}$ & VPP (\%) & $\mathrm{T}_{0}$ & $\mathrm{~T}_{1}$ & VPP (\%) \\
\hline \multirow[t]{3}{*}{ S. Francisco do Conde } & 12,3 & 3,8 & 84,2 & 2,1 & 0,6 & 71,4 \\
\hline & $(370)$ & & & $(370)$ & $(480)$ & \\
\hline & $(480)$ & & & & & \\
\hline \multirow[t]{2}{*}{ Boa Nova } & 8,7 & 1,8 & 79,3 & 6,7 & 1,3 & 80,6 \\
\hline & $(357)$ & $(3074)$ & & $(357)$ & $(3074)$ & \\
\hline \multirow[t]{2}{*}{ Torta } & 8,4 & 0,0 & 100,0 & 45,0 & 1,1 & 97,5 \\
\hline & (129) & $(278)$ & & $(129)$ & $(278)$ & \\
\hline
\end{tabular}

* $\mathrm{T}_{0}$ corresponde ao início e $\mathrm{T}_{1}$ ao final do estudo. Números entre parênteses corresponde ao tamanho da população do estudo; VPP = variação proporcional da prevalência percentual no período do estudo.

apenas para a população geral e masculina. Especificamente, entre os escolares (Tabela 2) o padrão foi semelhante ao observado na população geral, com VPP'e elevadas, nas áreas com ou sem o IEC/MC e valores máximos em Torta. Entre os indivíduos nessa mesma faixa

Tabela 2 - Prevalência da infecção por S. mansoni de acordo com o nível da carga parasitária, entre indivíduos de 7 a 14 anos, por área e ano do estudo.

\begin{tabular}{|c|c|c|c|c|c|c|}
\hline \multirow{3}{*}{ Área do estudo } & \multicolumn{6}{|c|}{ Nível da carga parasitária } \\
\hline & \multicolumn{3}{|c|}{1 a 4 ovos/lâmina } & \multicolumn{3}{|c|}{$>4$ ovos/lâmina } \\
\hline & $\mathrm{T}_{0}$ & $\mathrm{~T}_{1}$ & VPP (\%) & $\mathrm{T}_{0}$ & $\mathrm{~T}_{1}$ & VPP (\%) \\
\hline \multirow[t]{2}{*}{ S. Francisco do Conde } & 11,6 & 2,7 & 76,7 & 4,7 & 0,9 & 80,9 \\
\hline & (86) & (111) & & $(86)$ & (111) & \\
\hline \multirow[t]{2}{*}{ Boa Nova } & 11,7 & 4,4 & 62,3 & 9,5 & 3,9 & 58,9 \\
\hline & $(253)$ & $(923)$ & & (253) & (923) & \\
\hline \multirow[t]{2}{*}{ Torta } & 37,2 & 0,0 & 100,0 & 45,5 & 3,5 & 92,3 \\
\hline & (121) & (58) & & (121) & (58) & \\
\hline
\end{tabular}

${ }^{*} \mathrm{~T}_{0}$ corresponde ao inİcio e $\mathrm{T}_{1}$ ao final do estudo. Números entre parênteses corresponde ao tamanho da população do estudo; $\mathrm{VPP}=$ variação proporcional da prevalência percentual no período do estudo.

de idade, analisou-se o impacto diferencial por sexo (Tabela 3), o qual revelou pequenas diferenças indicativas de que os homens estariam apresentando resultados mais favoráveis do que as mulheres, especialmente em Boanova, área com IEC/MC. Vale ressaltar que em S. Francisco do Conde, foi muito baixa a queda relativa da prevalência de infecção de baixa carga parasitária entre mulheres, 28,4\%, em comparação ao sexo masculino, que obteve 100\% de redução de casos nessa mesma faixa de intensidade de infecção. Nota-se também uma tendência de maior redução de infectados com baixa carga parasitária, exceção para as mulheres em Boanova e S. Francisco do Conde. 
Santana VS, Teixeira MG, Santos CP, Andrade CAR. Efetividade do Programa de Comunicacão e Educação em Saúde no controle da infecção por S. mansoni em algumas áreas do Estado da Bahia. Revista da Sociedade Brasileira de Medicina Tropical 30:447-456, nov-dez, 1997.

\begin{tabular}{|c|c|c|c|c|c|c|}
\hline \multirow{3}{*}{ Área do estudo } & \multicolumn{6}{|c|}{ Nível da carga parasitária } \\
\hline & \multicolumn{3}{|c|}{1 a 4 ovos/lâmina } & \multicolumn{3}{|c|}{$>4$ ovos/lâmina } \\
\hline & $\mathrm{T}_{0}$ & $\mathrm{~T}_{1}$ & VPP (\%) & $\mathrm{T}_{0}$ & $\mathrm{~T}_{1}$ & VPP (\%) \\
\hline \multicolumn{7}{|l|}{$\begin{array}{l}\text { S. Francisco do Conde } \\
\text { masc }\end{array}$} \\
\hline masc & $\begin{array}{c}14 \\
(49)\end{array}$ & $\begin{array}{c}0,0 \\
(65)\end{array}$ & 100,0 & $\begin{array}{c}4,1 \\
(49)\end{array}$ & $\begin{array}{c}1,5 \\
(37)\end{array}$ & 63,4 \\
\hline fem. & $\begin{array}{l}8,1 \\
(37)\end{array}$ & $\begin{array}{l}5,8 \\
(52)\end{array}$ & 28,4 & $\begin{array}{c}5,4 \\
(37)\end{array}$ & $\begin{array}{c}0,0 \\
(52)\end{array}$ & 100,0 \\
\hline \multicolumn{7}{|l|}{ Boa Nova } \\
\hline masc & $\begin{array}{c}13,1 \\
(122)\end{array}$ & $\begin{array}{c}5,2 \\
(443)\end{array}$ & 60,3 & $\begin{array}{c}12,3 \\
(122)\end{array}$ & $\begin{array}{c}5,4 \\
(443)\end{array}$ & 56,1 \\
\hline fem. & $\begin{array}{c}9,2 \\
(131)\end{array}$ & $\begin{array}{c}4,0 \\
(480)\end{array}$ & 56,5 & $\begin{array}{c}6,8 \\
(131)\end{array}$ & $\begin{array}{c}2,7 \\
(480)\end{array}$ & 60,3 \\
\hline \multicolumn{7}{|l|}{ Torta } \\
\hline masc & $\begin{array}{l}38,0 \\
(71)\end{array}$ & $\begin{array}{l}0,0 \\
(27)\end{array}$ & 100,0 & $\begin{array}{l}45,1 \\
(71)\end{array}$ & $\begin{array}{c}3,7 \\
(27)\end{array}$ & 91,8 \\
\hline fem. & $\begin{array}{l}36,0 \\
(50)\end{array}$ & $\begin{array}{c}0,0 \\
(31)\end{array}$ & 100,0 & $\begin{array}{l}46,0 \\
(50)\end{array}$ & $\begin{array}{c}3,0 \\
(31)\end{array}$ & 93,5 \\
\hline
\end{tabular}

${ }^{*} \mathrm{~T}_{0}$ corresponde ao início e $\mathrm{T}_{1}$ ao final do estudo. Números entre parên
$\mathrm{VPP}$

\section{DISCUSSÃO}

Os dados deste estudo mostram que a implementação do Programa de Controle da Esquistossomose (PCE), com ou sem o componente IEC/MC, leva à redução da magnitude da infecção na população e que a presença desse programa, aparentemente, intensifica o impacto sobre a população em geral e entre as mulheres, qualquer que seja a faixa da carga parasitária. Esses resultados baseiam-se na comparação de Torta (área com programa IEC/MC) e S. Francisco do Conde (sem programa), desde que os achados relativos à Boanova devem ser vistos com reserva, devido à grande diferença no tamanho da população do estudo nos anos considerados na pesquisa. Essas características, certamente, explicam os afastamentos das tendências verificadas nessa localidade, em comparação com as duas outras áreas da pesquisa. Todavia, deve-se ressaltar que a carência de dados referentes ao processo de implementação das atividades IEC/MC, como cobertura, conteúdo ou abrangência das estratégias pedagógicas, ou ainda a outros fatores ambientais, sociais ou econômicos limita a atribuição de um papel mais relevante do IEC/MC em comparação às ações do PCE no controle da infecção por $S$ mansoni.

Não foram verificadas diferenças no padrão do impacto nas áreas com IEC/MC em relação à carga parasitária. Em ambas áreas, S. Francisco do Conde e Torta, as maiores quedas relativas da prevalência ocorreram nas faixas de menor carga parasitária, embora reduções significativas também fossem observadas no grupo de maior intensidade de infecção, com mais de quatro ovos por lâmina. Como a alta carga parasitária encontra-se positivamente associada ao desenvolvimento de formas clínicas ou quadros graves 7 , pode-se indicar que as intervenções contribuíram para minimizar os efeitos mais importantes da morbidade da esquistossomose.

É importante ressaltar que a análise desse estudo fica limitada, devido às diferenças nas faixas etárias selecionadas para os inquéritos realizados, em todas as três áreas, e também pela má qualidade da coleta ou armazenagem dos dados. Houve relatos de variações nos procedimentos de coleta no tempo, evidente no alto percentual de perdas de informações que chegou a atingir 40\% das observações. Todavia, esses problemas parecem não comprometer substancialmente as conclusões do estudo, desde que os achados, de um modo geral, confirmam resultados publicados anteriormente 7813 . É conhecida a complexidade do padrão de respostas populacionais com as intervenções típicas do PCE, fundamentadas no tratamento quimioterápico repetido, com recorrência dos níveis altos de morbidade, com uma tendência de retorno aos níveis iniciais10 22 que pode durar 2,8 a 3,2 anos 22 . Este fenômeno, já detectado anteriormente 225 , foi um dos principais motivos que levou o 
Santana VS, Teixeira MG, Santos CP, Andrade CAR. Efetividade do Programa de Comunicacão e Educação em Saúde no controle da infecção por S. mansoni em algumas áreas do Estado da Babia. Revista da Sociedade Brasileira de Medicina Tropical 30:447-456, nov-dez, 1997.

Ministério da Saúde, em 1989, a incluir o componente IEC/MC ao PCE, na perspectiva de que estas ações pudessem contribuir para dar uma maior sustentabilidade aos resultados que vinham sendo obtidos. Com base nos dados desse estudo, cujo intervalo de tempo foi cinco anos em Torta e Boanova, e de apenas um ano em S. Francisco do Conde, verificouse um declínio da prevalência da infecção em todas as áreas. Tal achado se encontra em aparente contradição com o conhecimento de que são mais comuns os padrões de recorrência da infecção, com tendência de retorno aos patamares iniciais. Ou seja, como não estão disponíveis dados para intervalos de tempo pelo menos anuais, não é possível traçar conclusões definitivas sobre a evolução da prevalência da infecção, no sentido da sua sustentabilidade temporal, aspecto crítico para a avaliação da efetividade desse programa.

Nota-se que, na população geral, o maior impacto ocorreu na localidade de Torta. Todavia, quando se trata especificamente de crianças e adolescentes, os dados são sugestivos de um impacto populacional menor das ações IEC/MC. Tais achados sugerem menor efetividade das estratégias pedagógicas aplicadas pelo IEC/MC entre os escolares, o que implica na necessidade de sua revisão na busca de melhor adequação às especificidades comportamentais desses grupos, conhecidos como de risco para essa enfermidade. Embora não tenham sido encontradas diferenças marcantes de acordo com o gênero, observou-se maior impacto entre os homens em S. Francisco do Conde, que pode expressar a atividade econômica e os perfis ocupacionais próprios de cada uma dessas áreas, que estão a merecer melhor investigação.

É de se notar a limitação desses dados, de natureza secundária, para a avaliação de um componente específico dos programas de controle, as ações educativas, especialmente das ações IEC/MC, sobre um, também específico, desfecho que é a magnitude de indicadores de infecção por $S$. mansoni. Os instrumentos de coleta de dados de rotina foram elaborados para subsídiar ações imediatas na população e não na perspectiva de avaliações específicas. Portanto, a introdução do IEC/MC não foi acompanhada de modificações no sistema de informações que permitissem a avaliação dessas práticas em particular. Além disso, a grande rotatividade de profissionais, as descontinuidades nos métodos de coleta dos dados e as alterações nos critérios de escolha das populações dificultam a comparabilidade dos resultados na perspectiva temporal. Perdas de informações, mesmo quando ocorrem aleatoriamente, são reconhecidas como capazes de introduzirem viézes em torno da hipótese nula. Ou seja, erros de informação e perdas de dados podem ter atenuado diferenças porventura existentes entre os indicadores estimados para as áreas onde as ações IEC/MC foram implementadas, quando comparadas com aquelas onde foram executadas apenas as atividades de rotina do PCE.

Pode-se afirmar que a precariedade dos dados não compromete totalmente os achados epidemiológicos, desde que algumas das tendências observadas são consistentes com o conhecimento existente. Os resultados desse estudo têm sua explicação e interpretação limitadas, também por não terem ficado disponíveis os achados do estudo etnográfico em uma perspectiva histórica dos conhecimentos e práticas da população, especialmente, em relação à esquistossomose e de outros aspectos do seu modo de vida. Os autores entendem que, apesar da importância das avaliações de impacto epidemiológico, estas são insuficientes ou pouco adequadas para avaliar intervenções de natureza subjetiva e pluriforme, que têm na abordagem qualitativa a sua metodologia de eleição. Ademais, os altos investimentos necessários para programas de controle de endemias, como o da esquistossomose, impõem a realização de avaliação de efetividade na perspectiva do custo, com pesquisas específicas para se estimar o custo/benefício e custo/efetividade que devem demarcar o futuro das pesquisas avaliativas e as decisões dos serviços de saúde.

\section{SUMMARY}

The Program for S. mansoni Control (PCE) has been developed in some areas of the State of Babia by the Fundação Nacional de Saúde (FNS). In 1989, activities on Information, Education, Comunication and Community Mobilization (IEC/MC) were initiated. This study evaluates the epidemiological impact of the IEC/MC, using a quasi-experimental study design strategy, comparing the prevalences of infection for S.mansoni in areas of IEC/MC and estimates of other areas. The data used were routinely collected by the local staff of the FNS. A decrease on the prevalence of $\mathrm{S}$. mansoni infection was found in all study areas, specially in those of 
Santana VS, Teixeira MG, Santos CP, Andrade CAR. Efetividade do Programa de Comunicacão e Educação em Saúde no controle da infecção por S. mansoni em algumas áreas do Estado da Bahia. Revista da Sociedade Brasileira de Medicina Tropical 30:447-456, nov-dez, 1997.

IEC/MC activities. Findings indicate that PCE activities are more effective among school-age individuals and male adults, although IEC/MC allows for higher epidemiological impact among women, reflecting the differences among the strategies. These findings point out the need for qualitative evaluation resarch, as well as cost benefit and cost effectiveness analyses, that are more appropriate for decision making processes.

Key-words: Schistosomiasi. S. mansoni. Control. Evaluation. Education Temporal tends.

\section{AGRADECIMENTOS}

Esse trabalho se tornou possível pela colaboração da Diretoria Regional da FNS da Bahia e dos seus funcionários do Distrito de Pernambués e Jequié. Foi também indispensável o apoio do Dr. Carlos Catão, que nunca deixou de acreditar na realização dessa pesquisa.

\section{REFERÊNCIAS BIBLIOGRÁFICAS}

1. Al-Madani AA. Schistosomiasis control in Saudi Arabia with special reference to the period 19831988. Public Health 4:261-266, 1991.

2. Amaral RS, Porto MAS. Evolução e situação atual do controle da esquistossomose no Brasil. Revista da Sociedade Brasileira de Medicina Tropical 27 (supl III):73-90, 1994.

3. Ashi J, Arfaat F, Jeffri M, Suwairy M. Progress achieved in the control of schistosomiasis in Saudi Arabia. The Journal of Tropical Medicine and Hygiene 92:27-31, 1989.

4. Bahia. Centro de Estatística e Planejamento. Informações básicas dos municípios baianos Recôncavo. CEI/CONDER, 224 pp.1994.

5. Barbosa FAS. Morbidade da Esquistossomose. Revista Brasileira de Malariologia e Doenças Tropicais ( $\mathrm{N}^{\circ}$ Esp)l:3-159, 1966.

6. Barbosa FS, Pinto RC, Souza OA. Control Schistosomiasis mansoni in a small North East Brazil community. Transactions of the Royal Society of Tropical Medicine and Hygiene 65:206213, 1971.

7. Bina JC. Influência da terapêutica específica na evolução da esquistossomose mansoni. Bahia. Tese de Mestrado, Faculdade de Medicina da Universidade Federal da Bahia, Salvador, 1977.

8. Bina JC. O tratamento específico como arma no controle da esquistossomose. Memórias do Instituto Oswaldo Cruz 87 (supl IV):195-202, 1992.
9. Bina JC. Estudo de variáveis que podem influenciar na evolução da esquistossomose mansônica: efeito da terapêutica específica na interrupção da transmissão. Bahia.Tese de Doutorado. Faculdade de Medicina da Universidade Federal da Bahia, Salvador, 1995.

10. Bina JC, Prata AR. Regressão da hepatoesplenomegalia pelo tratamento específico da esquistossomose. Revista da Sociedade Brasileira de Medicina Tropical 16:213-218, 1983.

11. Brasil. Conselho de Desenvolvimento Social. Programa Especial de Controle da Esquistossomose no Brasil (PECE) supl I, 1976.

12. Brasil. Ministério da Saúde/Superintendência de Campanhas de Saúde Pública (SUCAM) Normas gerais para o programa de Controle da Esquistossomose, Brasília, 1989.

13. Coura JR, Conceição MJ, Pereira JB. Morbidade da esquistossomose mansoni no Brasil III - Estudo evolutivo em uma área endêmica no período de dez anos. Memórias do Instituto Oswaldo Cruz 79:447-453, 1984.

14. Dalton PR. A sociological approach to the control of Schistosoma mansoni in St Lucia, West Indies. Bulletin of the Word Health.Organization, 54:587595, 1977.

15. Engels D, Donricimpa J, Gryseels B. Schistosomiasis mansoni in Burundi: progress in its control since 1985. Bulletin of the World Health Organization 71:207-214, 1993.

16. Kloos H. Human behavior, health education and schistosomiasis control: a review. Social Science and Medicine 40:1497-1511, 1995.

17. Costa MFFL, Guerra HL, Pimenta Júnior FG, Firmo JOA, Uchoa E. Avaliação do programa de controle da esquistossomose (PCE/PCDEN) em municípios situados na Bacia do Rio São Francisco, Minas Gerais, Brasil. Revista da Sociedade Brasileira de Medicina Tropical 29:117-126, 1996.

18. Costa MFFL, Rocha RS, Coura Filho P, Katz NA. A 13-year follow-up of treatment and snail control in an area endemic for Schistosoma mansoni in Brazil: incidence of infection and reinfection. Bulletin of the World Health Organization 71:197205, 1993.

19. Costa MFFL, Rocha RS, Leite MLC, Carneiro RC, Colley D, Gazzinelli G, Katz NA. Multivariate analysis of socio-demographic factors, water contact patterns and Schistosoma mansoni infection in na endemic area in Brazil. Revista do Instituto de Medicina Tropical de São Paulo 33:58-63, 1991. 
Santana VS, Teixeira MG, Santos CP, Andrade CAR. Efetividade do Programa de Comunicacão e Educação em Saúde no controle da infecção por S. mansoni em algumas áreas do Estado da Babia. Revista da Sociedade Brasileira de Medicina Tropical 30:447-456, nov-dez, 1997.

20. Marçal Júnior O, Patucci RMJ, Dias LCS, Hotta LK. Schistosomiasis mansoni in area of low transmission. I. Impact of control measures. Revista do Instituto de Medicina Tropical de São Paulo 33:83-90, 1991.

21. Moagel MA, Arfaa F, Abdulghani ME. The use of primary health care system in the control of schistosomiasis in the Riyadh Region of Saudi Arabia. Tropical Medicine and Parasitology 41:136-138, 1990.

22. Santana VS, Teixeira MGLC, Santos CCP. Avaliação das ações de controle da infecção esquistossomótica nas localidades de Cachoeira-Bahia, Bacia do Paraguaçu, 1982-1992. Revista da Sociedade Brasileira de Medicina Tropical 29:185-195, 1996.

23. SAS. Software Institute, version 6, first Edition. SAS Institute Inc., Cary, NC, USA, 1990.

24. Schall VT. Health education for children in the control of Schistosomiasis. Memórias do Intituto Oswaldo Cruz 82 (supl IV): 285, 1987.
25. Silveira AC. Controle da esquistossomose no Brasil. Memórias do Intituto Oswaldo Cruz 84 (supl I):91-104, 1989

26. Smith DH, Warren KS, Mahmoud AAF . Morbidity in Schistosomiasis mansoni in relation to intensity of infection: study of a community in Kisumu, Kenya. The American Journal Tropical Medicine and Hygiene 28:220-9, 1979.

27. Woolhouse MEJ. On the application of mathematical models of schistosome transmission dynamics. II. Control. (Review). Acta Tropica 50:189-204, 1992.

28. Zein AZ. Spontaneous reduction in Schistosoma mansoni infection in endemic communities of the lake Tana basin, North-Western Ethiopia. Transactions of the Royal Society of Tropical Medicine and Hygiene 83:656-658, 1989. 